\title{
LA FORMAZIONE POLITICO-CULTURALE DI ALCIDE DE GASPERI TRA TRENTO E VIENNA (1900-1918)
}

Ripercorre gli itinerari formativi di un importante personaggio è indubbiamente una ricerca ricca di stimoli, la cui suggestione maggiore scaturisce probabilmente soprattutto dalla consapevolezza di poter raggiungere, oltre l'individuo, l'ambiente in cui si plasmò la sua personalità, ampliando quindi la biografia individuale con una ricostruzione dei movimenti e delle tendenze presenti nella società in cui egli visse.

Sono queste impressioni che si adattano perfettamente ad un'indagine storica sulla giovinezza di Alcide De Gasperi, colui a cui spettò il compito di restituire all'Italia, dopo il ventennio di dittatura fascista, la guerra e la sconfitta, una solida democrazia, stabilità interna e un ruolo importante nella comunità internazionale, il politico e lo statista della storia dell'Italia repubblicana che godette del più alto prestigio in ambito internazionale.

De Gasperi fu diverso dagli altri politici italiani del dopoguerra ${ }^{1}$. Egli trascorse più della metà della sua vita - dal 1881 alla fine della prima guerra mondiale - all'interno della monarchia austro-ungarica e si formò a contatto con esperienze e modelli culturali e politici in gran parte sconosciuti negli ambienti italiani del suo tempo. Furono questi fattori che, se per un verso gli valsero incomprensioni e rimproveri da parte di contemporanei, di pubblicisti e storici, dall'altro contribuirono alla formazione di una personalità politica che seppe imporsi, come poche altre nell'Italia contemporanea, non solo nella politica interna ma anche e soprattutto in ambito internazionale.

De Gasperi nacque a Pieve Tesino nel Trentino asburgico, che da un punto di vista nazionale era un territorio compattamente italiano, inserito in una circoscrizione provinciale tedesco-italiana ma a maggioranza tedesca, il Tirolo, e in una monarchia multinazionale a guida tede-

1 A. Canavero, Alcide De Gasperi cristiano, democratico, europeo. Soveria Mannelli 2003 , VIII. 
sca $^{2}$. Agli inizi del ventesimo secolo il Trentino era una regione di confine contesa, luogo di contrasto, di conflitti etnici e a sfondo nazionale, ma al tempo stesso anche luogo di dialogo e di suggestioni profonde derivate dalla convivenza di due popoli e di due culture ${ }^{3}$. In uno dei suoi primi discorsi al Reichsrat a Vienna nel 1911 De Gasperi stesso sottolineò il prezioso ruolo di cerniera tra mondi culturali diversi svolto dai trentini e dai triestini: Sie müssen aber mindestens nicht vergessen, dass der Übersetzer Goethes und Schillers ein Trientiner war und dass die tridentinischen und triestinischen Literaten in der Zeit der Romantik zwischen der italienischen und der deutschen Literatur vermittelt haben; dass zu unserem Volkssplitter Borsieri, Prati, Rosmini, Dal Pozzo, Barbacovi, Martini und viele andere bekannte Namen gehören... ${ }^{4}$. E del resto, la stessa attività giornalistica e politica del giovane De Gasperi fu fortemente impregnata da un suo sentirsi e un voler essere mediatore tra il mondo italiano e quello germanico.

Quando nell'autunno del 1900 si iscrisse al primo anno della facoltà filosofica dell'Università di Vienna, De Gasperi aveva già conosciuto i fermenti nuovi che percorrevano il movimento cattolico trentino tra Ottocento e Novecento, movimento che per anni aveva avuto un indirizzo spiccatamente conservatore, come conservatore era anche il mondo contadino trentino vicino all'imperatore austriaco. Erano mutamenti che, per quanto legati alla specifica situazione trentina, andavano tuttavia collocati all'interno di ben più vaste trasformazioni sul piano europeo relative alla presenza politica dei cattolici nella società - a partire in particolare dall'enciclica Rerum Novarum di Leone XIII del 1891 -, trasformazioni che spinsero i cattolici a ricercare strumenti organizzativi e politici più adeguati per potersi inserire nella società e incidere con la loro presenza. A questo processo contribuirono anche fattori esterni ai mutamenti nella Chiesa e negli ambienti ecclesiastici, come in particolare

\footnotetext{
${ }^{2}$ Per uno sguardo d'insieme sul Trentino di quegli anni efr. Storia del Trentino V. L'età contemporanea 1803-1918, a cura di M. Garbari-A. Leonardi. Bologna 2003.

${ }^{3}$ A questo riguardo efr. le considerazioni svolte da A. Ara, Introduzione, in: Regioni di frontiera nell'epoca dei nazionalismi. Alsazia e Lorena / Trento e Trieste 1870-1914, a cura di A. Ara-E. Kolb. Bologna 1995, 8-9.

${ }^{4}$ Discorso tenuto da A. De Gasperi nel corso del dibattito sulla facoltà giuridica italiana, $20^{\circ}$ seduta della XXI sessione, 25 ottobre 1911, in: Stenographische Protokolle über die Sitzungen des Hauses der Abgeordneten des österreichischen Reichsrathes (=StPAH). Wien 1861-1918, XXI. Session, 20. Sitzung, 11711173.
} 
le pressioni ideologiche e politiche degli avversari, la volontà di vincere la supremazia liberale e le preoccupazioni antisocialiste ${ }^{5}$. Si era, in altri termini, di fronte al fenomeno dell'emergere delle masse, della loro politicizzazione e della conseguente apertura dei partiti e movimenti politici nei loro confronti.

Furono questi gli anni di grande sviluppo della cooperazione trentina e della nascita di diverse associazioni di matrice cattolica, tra cui nel 1896 quella della Società Agricola Operaia Cattolica di Trento animata da Celestino Endrici che di lì a qualche anno, nel 1904, sarebbe stato nominato dall'imperatore vescovo di Trento e avrebbe avuto grande influenza sul giovane De Gasperi ${ }^{6}$. Nel 1898 fu fondato il Comitato diocesano, l'anno seguente l'Associazione Universitaria Cattolica Trentina. Fu anche il momento di sviluppo della stampa cattolica trentina: dal 1896 iniziò la pubblicazione il giornale Fede e lavoro, mentre dal 1897 La voce cattolica fu trasformata da trisettimanale a quotidiano ${ }^{7}$.

Il 1896 era stato anche l'anno dell'attuazione della riforma elettorale Badeni che pur mantenendo un sistema elettorale che divideva gli elettori in corpi diversi, a seconda della loro fisionomia economico-sociale, affiancò una quinta curia eletta a suffragio universale alle quattro curie censitarie esistenti, favorendo alle elezioni del 1897 i cristiano-sociali austriaci ed i partiti nazionalisti tedeschi e cechi. In Trentino risultarono

${ }_{5}^{5}$ Sugli ambienti cattolici italiani e europei tra Ottocento e Novecento efr. I tempi della Rerum Novarum, a cura di G. De Rosa, Soveria Mannelli 2003; si veda inoltre A. Gambasin, Per uno studio sulle organizzazioni internazionali cattoliche, in: Genesi della coscienza internazionalista nei cattolici fra '800 e '900. Padova 1983, 1-16; Il cattolicesimo politico e sociale in Italia e Germania dal 1870 al 1914, a cura di E. Passerin d'Entrèves-K.Repgen. Bologna 1977. Sulle trasformazioni in ambito trentino cfr. A. Leonardi, Prime esperienze associative dei lavoratori cattolici trentini tra Ottocento e Novecento. Studi trentini di scienze storiche 58 (1979), 451-505; IDEM, La cooperazione: da un esordio difficile a uno sviluppo prorompente, in: Storia del Trentino (come in nota 2), 779-815; G. BETTA, Il movimento cattolico trentino fra ' 800 e '900. Organizzazione e ideologia. Materiali di lavoro 9/10 (1980), 1-143; S. VARESCHI, Il movimento cattolico trentino tra Ottocento e Novecento, in: Storia del Trentino (come in nota 2), $817-838$.

${ }^{6}$ Sull'operato di Celestino Endrici, il vescovo di chiari sentimenti italiani, e più ampiamente sui processi in corso nella società rurale trentina agli inizi del '900 efr. gli Atti del convegno trentino del 1991: Celestino Endrici (1866-1940) Vescovo di Trento. Trento 1992.

${ }^{7}$ G. Vecchio, De Gasperi e l'Unione Politica Popolare nel Trentino (1904-1914), in: De Gasperi e il Trentino tra la fine dell' 800 e il primo dopoguerra, a cura A. Canavero-A. Moioli. Trento 1985, 509-592, in particolare 512-515. 
eletti quattro cattolici nazionali, tra cui Enrico Conci, ed altrettanti liberali, segnando così un primo passo verso la perdita dell'egemonia liberale. Fu però solo alle nuove elezioni per il Reichsrat del dicembre 1900-gennaio 1901 che i cattolici in Trentino riuscirono a presentare candidature unitarie, eleggendo don Baldassare Delugan per la quinta curia, oltre a Bazzanella, Conci e Maffei ${ }^{8}$.

Proprio in questo periodo De Gasperi aveva iniziato i suoi studi a Vienna: aveva scelto come indirizzo fondamentale lingua e letteratura tedesca a cui affiancò la filologia classica. Gli studenti trentini in grande maggioranza frequentavano allora l'università in Austria, a Innsbruck, Graz e a Vienna: pochi erano invece gli iscritti nelle università del Regno d'Italia. ${ }^{9}$ De Gasperi seguì le lezioni di germanistica di Richard Heinzel, quelle del filosofo liberale Friedrich Jodl su Schopenhauer, ed inoltre quelle di filologia romanica di Adolfo Mussafia, figura simbolo per gli studenti italiani dell'impero, membro dell'Herrenhaus e delle più prestigiose accademie e istituzioni italiane, e di Mayer-Luebke ${ }^{10}$. Durante gli studi universitari De Gasperi ebbe una borsa di studio governativa, un Nicolaihaus Stipendium, che ammontava a 120 corone all'anno, concessa dalla k.k. Statthalterei di Innsbruck e abitò in una stanza nel Porzellaneum, lo studentato ancora oggi esistente nella Porzellangasse al numero 30; negli ultimi due semestri si trasferì nella Lazarethgasse al numero $8^{11}$.

${ }^{8}$ Sugli sviluppi politici nell'ambito della monarchia efr. H. Rumpler, Eine Chance für Mitteleuropa: bürgerliche Emanzipation und Staatsverfall in der Habsburgermonarchie (Österreichische Geschichte 1804-1914). Wien 1997, 488-515; IDEM, Parlament und Regierung Cisleithaniens 1867 bis 1914, in: Die Habsburgermonarchie 1848-1918, VII. Verfassung und Parlamentarismus, a cura di H. RunPLER-P. Urbanitsch. Wien 2000, 667-894, in particolare 831-894; nello stesso volume L. Höвelt, Parteien und Fraktionen im cisleithanischen Reichsrat, 895-1006, in particolare 942-996. Sui risultati elettorali in Trentino efr. Vессніо (come in nota 7), 515-516; M. Garbari, Aspetti politico-istituzionali di una regione di frontiera, in: Storia del Trentino (come in nota 2), 13-164, in particolare 117-139. Su Conci efr. M. Ghezzer, L'attività politica di Enrico Conci dal 1891 al 1918 nelle carte dell'archivio Enrico e Elsa Conci. Archivio Trentino di Storia contemporanea 1 (1995), 51-75.

${ }^{9}$ Cfr. S. Benvenuti, De Gasperi e l'ambiente studentesco, in: De Gasperi e il Trentino tra la fine dell'800 e il primo dopoguerra, a cura A. Canavero-A. Moioli. Trento 1985, 237-262; S. MalfÈr, Studenti italiani a Vienna, Graz e Innsbruck, 1848-1918. Il Politico 3 (1985), 493-508.

${ }^{10}$ Wien, Archiv der Universität, Rigorosenprotokoll e Rigorosenakt PN 1844 di Alcide De Gasperi.

11 Wien, Archiv der Universität, Nationalien/Inskriptionsblätter, Philosophische Fakultät, Wintersemester 1900/01-Sommersemester 1904. 
Si laureò nel luglio 1905 discutendo una tesi di laurea sui rifacimenti tedeschi della favola drammatica di Carlo Gozzi I pitocchi fortunati ${ }^{12}$.

La Vienna dove De Gasperi soggiornò da studente per quattro anni era la Vienna di fine secolo, ormai giunta alla vigilia del tramonto politico, ma che fu proprio allora uno dei centri più creativi e dei laboratori più innovativi e fecondi della cultura europea. Era la capitale che rifletteva più compiutamente di ogni altra città asburgica l'anima multiforme e plurinazionale della monarchia, al cui interno si intrecciavano e si arricchivano reciprocamente stimoli culturali provenienti da lingue e culture diverse. Ma era anche la capitale dello Stato multinazionale che, incapace di trasformarsi, era ormai prossimo alla dissoluzione. Era al tempo stesso la Vienna amministrata dalla innovativa politica comunale del borgomastro Karl Lueger, capo carismatico del movimento cristianosociale, movimento che proprio nei primi anni del '900, dopo essere stato un fenomeno soprattutto della politica comunale viennese, si diffuse negli altri centri austriaci, strappando il predominio ai conservatori catto$\operatorname{lici}^{13}$.

Si aprì proprio in questi anni un periodo intensissimo e importante per la formazione di De Gasperi, durante il quale le sue esperienze viennesi si intrecciarono strettamente con quelle trentine. Iniziò allora la sua attività di mediatore e interprete fra i movimenti cattolici in Italia e in Austria attraverso i suoi articoli nella stampa trentina, in particolare nel giornale La voce cattolica che nel 1906 cambiò testata e divenne Il Trentino, e in quella viennese, nel quotidiano cristiano-sociale Reichspost, approfondendo al contempo il suo impegno diretto nell'ambito dell'associazionismo cattolico e del nascente Partito Popolare Trentino. Entrambe le attività sono documentate e ricostruibili proprio tramite i numerosissimi articoli e note sulla stampa quotidiana, in cui De Gasperi inquadrava e raccontava eventi grandi e piccoli, ma anche tramite gli appunti che egli preparava per le sue conferenze, raccolti in parte nel suo archivio

12 A. De Gasperi, Die Glücklichen Bettler von Carlo Gozzi und ihre deutschen Bearbeitungen. Non è stato finora ritrovato alcun esemplare della dissertazione.

${ }^{13}$ Sulla Vienna tra fine Ottocento e inizi Novecento efr. C. E, Schorske, Fin-desiècle Vienna. Politics and Culture. New York 1980, trad. ital. Milano 1981; cfr. inoltre Annali/Jahrbuch Isig, XXVI (2000), 255-390, con una sezione dedicata al tema Vienna intorno al 1900 / Wien 1900. Su Karl Lueger e i cristiano-sociali cfr. J. W. Boyer, Political Radicalism in late Imperial Vienna. Origins of the Christian Social Movement 1848-1897. Chicago 1981, in particolare 184-521; IDEM, Culture and Political Crisis in Vienna. Christian Socialism in Power, 1897-1918. Chicago 1995. 
privato, fino ad ora conservato dalla famiglia e che verrà depositato negli Archivi storici delle Comunità europee presso l'Istituto Universitario Europeo di Firenze ${ }^{14}$. Altre fonti sono andate distrutte in due guerre mondiali e nel periodo intermedio della repressione fascista. Bisogna inoltre tenere presente che c'è stata non poca cautela politica, in particolare a partire dal 1907, sia da parte dei cristiano-sociali viennesi che dei cattolici trentini, accusati spesso di scarso sentimento nazionale, nel parlare apertamente dei loro legami ideologici, politici e privati ${ }^{15}$.

Agli esordi di De Gasperi come oratore in occasioni pubbliche nei primi mesi del 1901 parlando a Civezzano per la Società Agricola Operaia Cattolica, fecero poi seguito il suo 'giro di propaganda' nel settembre 1903 nel Vorarlberg, dove c'erano migliaia di immigrati trentini, e soprattutto le conferenze che egli tenne tra il 1904 e il 1905 per gli operai italiani immigrati a Vienna. I temi che egli affrontava erano molteplici: dagli sviluppi della democrazia cristiana nei vari paesi d'Europa, dimostrando come il Trentino non facesse che seguire le orme dei più avanzati, alle origini del socialismo e ai moniti del pontefice contro i suoi errori, dai vari sistemi sociologi da Platone a Karl Marx alla Rerum novarum ${ }^{16}$. Si delineava il profilo del giovane leader politico che nel confronto col socialismo sottolineava l'aspetto militante della sua proposta democratico-cristiana - conveniva scuotere ed eccitare, ed è quanto che si è tentato ora ${ }^{17}$ - e lasciava intuire la forte influenza che in lui già esercitava il linguaggio e lo stile di Lueger.

Ma accanto al De Gasperi conferenziere e organizzatore sociale degli operai e del 'popolo' vi era il De Gasperi presidente dell'Associazione

${ }^{14}$ Della foltissima produzione pubblicistica di De Gasperi dal 1902 al 1915 è stata pubblicata una raccolta di articoli apparsi su Fede e lavoro, La voce cattolica e Il Trentino: A. De Gasperi, I cattolici trentini sotto l'Austria. Antologia degli scritti dal 1902 al 1915 con discorsi al Parlamento austriaco, 2 voll. Roma 1964.

15 A. Wandruszka, De Gasperi e il movimento cristiano in Austria, in: De Gasperi e il Trentino tra la fine dell' 800 e il primo dopoguerra, a cura A. CAnavero-A. Moioli. Trento 1985, 225-236.

${ }^{16}$ Nell' Archivio De Gasperi, Austria I, sono conservati alcuni manoscritti e testi a stampa delle conferenze tenute da De Gasperi durante il periodo universitario, tra cui un discorso Sui doveri dell'ora presente di fronte ai socialisti, e una conferenza su Sguardo storico di sociologia, da cui emerge il suo interesse per gli scritti di Karl Marx e in particolare per la sua teoria dell'evoluzione sociale. Si veda anche F. Rasera, Lotta al socialismo e origini del movimento cattolico, in: De Gasperi e il Trentino tra la fine dell' 800 e il primo dopoguerra, a cura di A. Canavero-A. Moioli. Trento 1985, 419-463, in particolare 425.

${ }^{17}$ Dopo un giro di propaganda nel Vorarlberg. La voce cattolica (3 ottobre 1903). 
Universitaria Cattolica Trentina, che teneva discorsi alle serate dell'Unione Accademica Cattolica Italiana di Vienna affrontando vari argomenti, come Il compito dei giovani e La democrazia dei fatti, oppure soffermandosi sulla libertà di stampa o ancora sui rapporti tra Schiller e Carlo Gozzi ${ }^{18}$.

Proprio tramite le associazioni studentesche cattoliche viennesi, tra cui in particolare la Norica, De Gasperi entrò in contatto con Franz Hemala, il futuro organizzatore del movimento sindacalista cattolico in Austria, incontrò il docente di teologia dogmatica Ernst Commer, con cui fece un viaggio a Roma nel marzo del 1902, strinse amicizia con Friedrich Funder, il direttore prima della Reichspost e poi dopo la seconda guerra mondiale del settimanale Die Furche, un'amicizia che continuerà fino alla morte di De Gasperi ${ }^{19}$. Se ne conoscono alcuni episodi, su cui ci si sofferma brevemente perché contribuiscono a sottolineare il ruolo di mediatore tra gli ambienti italiani e trentini in particolare e quelli austriaci svolto da De Gasperi. Nel maggio del 1905, quando De Gasperi era ancora studente a Vienna, Funder si rivolse a lui preoccupato per la sorte del partito cristiano-sociale, al cui interno circolava da tempo la notizia che fosse prossimo un intervento del Vaticano che ne avrebbe limitato l'attività sulla base delle norme che erano state emanate nei confronti del movimento democratico-cristiano italiano. Funder chiedeva tramite De Gasperi che il vescovo di Trento ricevesse in via informativa e con la massima discrezione Albert Gessmann che stava a capo dell'organizzazione del partito cristiano-sociale austriaco. Nel mentre compio il mio

${ }^{18}$ Un elenco dei temi da lui affrontati è riassunto da M. Demattè, Alcide Degasperi all'alba del XX secolo. Trento 1962, 48. Alcuni discorsi sono stati pubblicati sulla stampa, si veda in particolare Il compito dei giovani. La voce cattolica (16 febbraio 1903); Riformiamo noi stessi. La voce cattolica (29 gennaio 1904).

19 Sulla Norica efr. B. Moser, Die katholisch akademische Studentenverbindung Norica in Wien 1883-1938. Versuch einer Strukturanalyse. Diss., Wien 1983, 76-198. Assieme al fratello Luigi Mario (morto nel 1906 a soli 23 anni) Alcide De Gasperi contribuì alla traduzione in italiano dell'opera di Commer Die Kirche in ihrem Wesen und Leben, pubblicata a Vienna nel 1904. Sul viaggio a Roma che costituì un'esperienza importante per la formazione dello studente trentino, durante il quale ebbe tra l'altro modo di incontrare il promotore in Italia del movimento democratico cristiano Romolo Murri, e sull'influenza di Ernst Commer sulla formazione di De Gasperi cfr. L. Bedeschi, Il giovane De Gasperi e l'incontro con Romolo Murri. Milano 1974, 27-84; A. Zambarbieri, Appunti sulla formazione spirituale del giovane De Gasperi, in: De Gasperi e il Trentino tra la fine dell'800 e il primo dopoguerra, a cura di A. Canavero-A. Moioli. Trento 1985, 379-418. 
dovere di mediatore, - scriveva De Gaspari al termine della lettera con cui informava il vescovo Endrici dei timori e della richiesta di Funder - $m i$ permetto d'esprimere la mia umile opinione nel senso che, fondate o no le premesse, un simile colloquio avrebbe però grande valore per la causa cattolica, e, visto il futuro che aspetta in Austria al partito c.s., può porre V. A. in grado di giovare alla Chiesa ${ }^{20}$. Non si sa quale sia stata, se c'è stata, la risposta di Endrici.

Vi fu poi il noto sondaggio esplorativo di De Gasperi, allora deputato al Reichsrat, presso Funder prima dell'ingresso dell'Italia nella prima guerra mondiale, quando si erano diffuse le voci di una possibile cessione del Trentino all'Italia in cambio della neutralità di quest'ultima e durante il quale De Gasperi avrebbe parlato degli interessi che spingevano la gente trentina a restare unita all'Austria, col probabile intento di ottenere garanzie sul mantenimento delle autonomie godute dalla sua regione sotto l'impero austro-ungarico. Di questo colloquio Funder stesso riferì nelle sue memorie pubblicate nel 1952, senza però esplicitare il nome del deputato italiano di cittadinanza austriaca che gli aveva fatto visita, per non nuocere a De Gasperi a capo del governo italiano ${ }^{21}$.

De Gasperi e Funder rimasero in contatto anche durante gli anni Venti quando, dopo la fuga dai fascisti, l'arresto, il carcere e la concessione della grazia, il politico trentino riuscì a trovare un impiego alla Biblioteca Vaticana, dove rimase dal 1929 al 1943. Nei primi anni Venti De Gasperi scriveva a Funder ringraziandolo per l'invio di una relazione dettagliata, senza però specificarne il contenuto ${ }^{22}$. All' inizio del marzo 1938 Funder era a Roma e fece visita a De Gasperi in Vaticano ${ }^{23}$. Si rividero poi per l'ultima volta durante un altro viaggio a Roma di Funder nell'autunno del 1953, durante il quale De Gasperi espresse a Funder il desiderio che fosse mantenuto l'anonimato sul deputato del parlamento austriaco che gli aveva fatto visita nell'autunno del 1914. De Gasperi era

20 Trento, Archivio della Curia Arcivescovile, Acta Episcopi Endrici, n.238, lettera di Alcide De Gasperi al vescovo Endrici, Vienna, 22 maggio 1905. La lettera è stata integralmente pubblicata da U. CorsinI, Il colloquio Degasperi-Sonnino. I cattolici trentini e la questione nazionale. Trento 1975 , 150-153, nota 41.

${ }^{21}$ F. Funder, Vom Gestern ins Heute. Aus dem Kaiserreich in die Republik. Wien-München 1953, 527-528.

${ }^{22}$ Lettera di Alcide De Gasperi a Friedrich Funder, Roma 28 settembre, senza anno, ma su carta intestata della Camera dei Deputati, di cui De Gasperi fu membro dal 1921 al 1926, conservata presso la famiglia Funder a Baden. Ringrazio il Prof. Dr. Ernst Bruckmüller per avermi segnalato la lettera e avermi messo in contatto con la famiglia Funder.

${ }^{23}$ F. Funder, Persönliche Erinnerungen. Die Furche (28 agosto 1954). 
probabilmente ancora memore dei duri attacchi, che avevano per oggetto la sua attività parlamentare a Vienna, subiti durante l'ultima e difficile campagna elettorale della primavera 1953 in particolare da parte dello MSI. Funder acconsentì, inviando anche una breve dichiarazione a tal proposito all'ambasciatore austriaco a Roma, Johannes Schwarzenberg. Ma subito dopo la scomparsa di De Gasperi nell'agosto 1954, essendo scoppiate nuove polemiche sul nome del deputato che gli aveva fatto visita durante la guerra, Funder scrisse a Schwarzenberg che gli sembrava fosse ormai giunto il momento di sollevare l'anonimato e di fornire una corretta versione dei fatti. Da questa sua convinzione scaturì probabilmente la decisione di rispondere ad una lettera di Giulio Andreotti dell'ottobre 1954, che lo pregava di fornirgli informazioni sull'attività di De Gasperi prima del 1919 per la biografia che si stava accingendo a scrivere, raccontando anche di quel suo colloquio durante la prima guerra mondiale col deputato Alcide De Gasperi24

Verso la fine del 1901 De Gasperi entrò a far parte di un "Comitato di azione pro università" a rappresentare gli studenti cattolici trentini a Vienna in un organismo unitario, comprendente anche un esponente liberale e uno socialista, che si batteva per l'istituzione di un'università italiana all'interno della monarchia ${ }^{25}$.

La cosiddetta 'Universitätsfrage' è stata tra il 1866 e il 1914 il problema più fortemente sentito e vissuto sia da tutti gli austro-italiani che nelle sue ripercussioni all'interno del Regno d'Italia ${ }^{26}$. Gli italiani si trovavano allora a condividere la sorte degli altri gruppi nazionali che in Austria non possedevano una propria università e iniziarono a rivendicare l'istituzione di un ateneo italiano a Trieste, la maggiore città italiana dell'Austria. Fino alla fine dell'800 i vari governi riuscirono a mantenere

${ }^{24} \mathrm{Si}$ vedano le lettere di Johannes von Schwarzenberg a Karl Gruber (8 giugno 1953), di Schwarzenberg a Friedrich Funder (20 novembre e 18 dicembre 1953, 8 settembre 1954), di Funder a Schwarzenberg (23 dicembre e 30 dicembre 1953, 3 settembre 1954), la lettera di Giulio Andreotti a Funder (21 ottobre 1954) e la risposta di Funder, Wien, Österreichisches Staatsarchiv, Allgemeines Verwaltungsarchiv, Nachlass Friedrich Funder, E/1781:50.

${ }^{25}$ Cfr. F. Rasera (come in nota 16), 429-430.

${ }^{26}$ Sulla questione universitaria efr. A. ARA, La questione dell'università italiana in Austria, in: IDEM, Ricerche sugli austro-italiani e l'ultima Austria. Roma 1974, 9-140; A. Schusser, Zur Entwicklung der italienischen Universitätsfrage in Österreich (1861-1918). Untersuchungen über das Verhalten von Regierung und Parlament zur Schaffung einer italienischen Rechtsfakultät. Diss., Wien 1972; Per l'università italiana in Austria. Carteggio trentino 1898-1920, a cura di V. Calì. Trento 1990. 
la questione più o meno in ambito amministrativo, oscillando tra rifiuti e dilazioni. Ma nel 1901 il governo Körber promise che avrebbe fondato a Wilten, presso Innsbruck, un'autonoma facoltà italiana di giurisprudenza. L'inaugurazione sarebbe dovuta avvenire nel novembre del 1904, ma il clima politico della città era incandescente: già da alcuni anni, da quando erano state istituite due cattedre in lingua italiana nell'ambito della facoltà di legge, si verificavano incidenti tra studenti italiani e studenti tedeschi. La sera della vigilia scoppiarono violenti disordini e tumulti - i cosiddetti 'fatti di Innsbruck' - tanto che il governo dovette rinunciare all'idea di mantenere nella capitale tirolese una facoltà giuridica italiana. De Gasperi, che si era recato a Innsbruck per partecipare all'inaugurazione con altri studenti italiani tra cui anche Cesare Battisti, fu coinvolto nei tumulti, fu arrestato e chiuso per un certo periodo nel carcere di Innsbruck ${ }^{27}$. Nel corso degli anni furono poi proposte nuove sedi per la facoltà italiana di giurisprudenza, tra cui anche Rovereto, Trento, Vienna e nel 1913 Trieste, ma il progetto non andò mai in porto e fu poi del tutto vanificato dallo scoppio della guerra.

Quello che qui però ci interessa è sottolineare la posizione di De Gasperi rispetto al problema dell'università italiana, soprattutto perché aiuta a comprendere la sua posizione rispetto alla questione nazionale e come egli viveva quest'ultima all'interno dello Stato plurinazionale degli Asburgo. Nel 1901 De Gasperi aveva dato la sua adesione alla richiesta di un ateneo italiano a Trieste, adesione ribadita anche l'anno seguente. Però di fronte alla richiesta socialista e irredentista che pretendeva $o$ Trieste o nulla, egli iniziò a propendere per una via possibilista più flessibile, dichiarandosi anche a favore della soluzione trentina ${ }^{28}$. Ma da deputato appena eletto al Reichsrat egli prese decisamente posizione, esprimendosi in quella sede il 25 ottobre del 1911 in un discorso a favore dell'istituzione di una facoltà giuridica ed economica in lingua italiana a Vienna, dopo che i deputati Erler di Innsbruck e Wastian di Marburg si erano dichiarati contrari: Ich stelle fest, dass gerade vom österreichischen Standpunkte aus die Fakultät eine Notwendigkeit, ein kategorischer Imperativ geworden ist. ... Man hat gesagt, die Fakultät wird der Heranbildung der irredentistischen Intelligenz dienen. Im Gegenteil, meine Herren, nicht die Fakultät, sondern die Fakultätsfrage schafft und verschärft die Erbitterung in unserer Jugend. ... Will man aber damit vielleicht sagen, dass die Fakul-

\footnotetext{
${ }^{27}$ Sui fatti di Innsbruck e in particolare sul coinvolgimento di De Gasperi efr. A. Schusser (come in nota 26), 187-195.

${ }^{28}$ Cfr. A. Schusser (come in nota 26), 224.
} 
tät nur die Kadettenschule unserer Bourgeoisie sein wird, so liegt auch dieser Behauptung eine krasse Unkenntnis unserer Verhältnisse zugrunde. Gerade das Gegenteil ist wahr. Die Söhne unserer Bourgeoisie besitzen auch jetzt die Mittel sich anderen Studien zu widmen, entweder in Rom, Florenz, Leipzig oder Berlin zu studieren. Die Fakultät dagegen soll auch den Bauernsöhnen die Möglichkeit bieten, billig und bequem in der eigenen Heimat zu studieren. Wer also gegen die Fakultät ist, ist nicht gegen die sogenannte Irredenta, sondern sündigt an den Söhnen eines Volkes, welches nach dem bekannten Auspruche unseres Kaisers selbst, ..., österreichischer gesinnt ist als man glauben konnte. E continua spiegando la differenza per lui fondamentale tra l'irredentismo generico con un fondamento storico, che non era altro che il corollario del principio nazionalistico, welches nur einheitlich nationalgebildete Staaten annimmt, e il ben diverso sentimento der geistigen Kulturgemeinschaft mit unserer italienischen Nation, die Begeisterung für unsere Geschichte und für unser Volkstum, che potevano essere difesi anche nel quadro istituzionale dell'impero asburgico ${ }^{29}$. Difensore quindi dei diritti nazionali italiani senza però giungere alla rivendicazione irredentista dell'annessione all'Italia. Gli interessi e i problemi che tenevano occupato De Gasperi erano quelli sociali e religiosi, a cui subordinava l'ideale di patria nel senso risorgimentale del termine. Come ha giustamente sottolineato Umberto Corsini: La sconfitta e il crollo dell'Impero lo trasferiva non da una nazione ad un'altra, ma da uno Stato ad un altro; e all'interno del secondo, l'Italia, egli riprese a lottare per diffondere, difendere e affermare le sue idealità religiose sociali, come ed ugualmente aveva fatto nell'impero asburgico ${ }^{30}$. In De Gasperi era preminente il senso dello Stato su quello della nazione, non provò alcun conflitto morale nel rimanere fedele cittadino della duplice monarchia e al tempo stesso sentendosi italiano e agendo come tale. Anche la battaglia da lui condotta contro gli Alldeutschen fu lotta 'culturale' e religiosa prima che nazionale, in difesa del cattolicesimo legato alla latinità, contro il protestantesimo che egli vedeva legato invece al germanesimo. Del resto sono note le molte riserve di De Gasperi nei confronti del Regno d'Italia, del suo indirizzo politico e dei suoi governi di un laicismo che lui non poteva condividere.

Appena laureato nell'estate del 1905 De Gasperi fu posto dal vescovo Endrici alla direzione del quotidiano La voce cattolica, che di lì a qualche mese, nel marzo 1906, avrebbe cambiato testata in Il Trentino e continuò

\footnotetext{
29 Discorso De Gasperi (come in nota 4).

30 U. Corsini (come in nota 20), 9 .
} 
ad uscire sino alla vigilia della dichiarazione di guerra dell'Italia all'Austria-Ungheria, quando lasciò spazio al giornale ufficiale $I l$ risveglio austriaco.

Gli anni che precedettero il ritorno di De Gasperi a Vienna come deputato al Reichsrat nel 1911, lo videro sempre diviso tra l'attività politica in Trentino e i contatti e gli scambi con gli ambienti cattolici austriaci e tedeschi e dei democratici cristiani italiani, mentre si intensificava anche il suo interessamento per le attività dei cattolici belgi, olandesi, russi, polacchi e sloveni.

Va sottolineata in particolare la sempre più frequente e attiva partecipazione di De Gasperi a congressi 'internazionali' che contribuirono a plasmare la sua personalità politica così aperta all'internazionalismo. Nell'estate 1903 partecipò come giornalista e rappresentante della Federazione trentina delle Società Agricole Operaie Cattoliche al congresso della lega generale austriaca dei lavoratori cristiani. Nel suo intervento De Gasperi si dimostrò deciso sostenitore dell'unione professionale che doveva superare le delimitazioni nazionali: Anche $i$ democratici cristiani italiani sono dell'opinione che solo una forte organizzazione dei lavoratori, a qualunque nazionalità appartengano, può servire veramente ai loro interessi. Le questioni nazionali devono cessare di fronte ai bisogni urgenti dei lavoratori ${ }^{31}$.

Appena laureato De Gasperi partecipò al quinto congresso dei cattolici austriaci che si tenne a Vienna nel novembre 1905, a ben nove anni di distanza dal precedente di Salisburgo a causa delle spaccature creatosi tra conservatori e cristiano-sociali. Al giovane politico trentino fu affidato un ruolo di prestigio nell'ambito della presidenza del congresso: toccò a lui infatti rappresentare le nazionalità non tedesche nel corso delle trattative per stabilire uno statuto provvisorio dell'organizzazione generale di tutti i cattolici entro i confini austriaci ${ }^{32}$. Nel 1907 De Gasperi prese parte anche al sesto congresso dei cattolici in Austria ${ }^{33}$.

Quello stesso anno De Gasperi partecipò anche al Katholikentag di Würzburg, durante il quale conobbe August Pieper, animatore del Volksverein e vecchio condiscepolo di Endrici, Karl Sonnenschein e Karl Muth. Tra gli esempi che De Gasperi tenne senz'altro presenti nell'organizzare l'attività dei suoi conterranei vi fu anche quello rappresentato dal Zentrum e soprattutto dal Volksverein in Germania. Nel primo quindicennio

\footnotetext{
${ }^{31}$ Congresso della lega gen.le austriaca dei lavoratori cristiano-sociali. La voce cattolica (2 luglio 1903).

${ }^{32}$ Cfr. G. Vecchio (come in nota 7), 531.

${ }^{33}$ Il sesto congresso dei cattolici austriaci. Il Trentino (20 e 22 novembre 1907).
} 
del nuovo secolo, infatti, il Volksverein appariva come una solidissima ed efficace struttura sociale ed educativa cattolica. Mönchen-Gladbach, la città in cui aveva sede il Volksverein era persino meta di viaggi di studio. Corsi d'istruzione e formazione, leghe sindacali, unioni professionali, attività editoriali etc. contribuivano a raccogliere attorno al Volksverein una massa enorme di iscritti ${ }^{34}$.

I rapporti personali e soprattutto la partecipazione di De Gasperi ai problemi austriaci e viennesi non si interruppero alla fine del periodo universitario per venire ripresi poi solo dopo la sua elezione al Reichsrat. Lo dimostrano ancora una volta gli articoli de $I l$ Trentino che in particolare negli anni 1907 e 1910 sono spesso dedicati ai cristiano-sociali e alle questioni politiche viennesi.

Un'analisi particolarmente attenta dei cristiano-sociali fu pubblicata nel marzo del 1907, in occasione del congresso del partito ${ }^{35}$. Veniva posto in risalto il carattere di vera e propria democrazia cristiana dei cristianosociali: entro al piccolo nucleo degli antisemiti viennesi spuntava, cresceva il fiore della riforma sociale cristiana... Certo, riconosceva Il Trentino, la democrazia cristiana austriaca aveva assunto dei connotati tutti particolari, giungendo anche a contraddizioni e confusioni nel programma politico, specie sul problema delle nazionalità. Ma, in definitiva, il partito cristiano-sociale sembrava potere incarnare quell'immagine politica vagheggiata da De Gasperi: Non promesse vane, non dottrinarismo che divide e stanca, ma politica pratica congiunta a quel tanto di disegno programmatico che ̀̀ indispensabile per l'omogeneità degli intenti e per non cadere nell'empirismo gretto.

Emergeva chiaramente l'ammirazione per Karl Lueger: si divulgava la sua immagine di 'campione' e di 'bandiera' del cattolicesimo militante antisemita e antiliberale ${ }^{36}$. Ampie tracce di quell'antisemitismo luegeriano che si proponeva di rompere la potenza del giudaismo che congiura a danneggiare moralmente ed economicamente $i$ popoli cristiani si ritrovavano del resto anche nel cattolicesimo trentino a cavallo del secolo, fino alla concezione di un antisemitismo ragionevole, rispettoso delle leggi della giustizia cristiana come un dovere di ogni cattolico ${ }^{37}$. Erano gli anni in cui in

${ }^{34}$ A. D[EGAsperi], Il congresso cattolico germanico. Il Trentino (26 agosto 1907).

${ }_{35}$ R., Il congresso dei cristiano-sociali. Il Trentino (15 marzo 1907).

36 Cfr. G. Vecchio (come in nota 7), 538.

37 Il sionismo in Austria. La voce cattolica (31 maggio-1 giugno 1902). Per questi aspetti cfr. F. RASERA (come in nota 16), 431-433; IDEM, Degasperi e il socialismo (1901-1921). Materiali di lavoro 3 (1984), 3-45; A. Esposito-D. Quaglioni, Processi contro gli ebrei di Trento (1475-1478), vol. I. I processi del 1475. Padova $1990,5-6$. 
Trentino fu ripresa l'accusa agli ebrei di omicidio rituale legata alla storia di S. Simonino. Il parroco di S. Pietro, la chiesa del culto di S. Simonino, aveva pubblicato proprio nel 1902 due volumi sulla storia di Simonino e De Gasperi doveva conoscerli ed esserne stato in un qualche modo suggestionato, tanto da tenere nello stesso anno una conferenza su quel tema ai trentini residenti a Vienna ${ }^{38}$. Ancora nel 1906, nel corso di un contradditorio coi socialisti il cui resoconto occupa la prima pagina del suo giornale, De Gasperi ribadiva gli stessi temi accusando i sindacati tedeschi di ispirazione socialista di essere ormai dominati dagli ebrei: noi non siamo contro gli ebrei perché d'altra religione e d'altra razza, ma dobbiamo opporci ch'essi coi loro denari mettano il giogo degli schiavi sui cristiani $^{39}$.

Vi era quindi in Trentino un ambiente favorevole all'influenza delle concezioni cristiano-sociali viennesi. Quello che in particolare colpiva De Gasperi e i popolari trentini era però la mobilitazione permanente impressa alla base sociale del partito viennese: la parola magica [era] agitazione (propaganda) coraggiosa, instancabile, senza posa ${ }^{40}$. Lueger un abilissimo inventore di ogni mezzo che potesse servire per coinvolgere le grandi masse viennesi, che poi seppe usare abilmente sul piano politico. Ovviamente De Gasperi era consapevole della tipicità viennese del partito di Lueger, ne colse così solo i segnali, cercando di interpretarli e di riutilizzarli nel contesto trentino.

Le varie campagne elettorali organizzate da De Gasperi tra il 1907 e il 1911 ne furono però chiaramente influenzate, a partire da quella per le elezioni al Reichsrat del 1907, che avvennero sulla base della nuova legge elettorale del 1906 che introduceva il suffragio universale uguale e diretto e aboliva le antiquate curie. De Gasperi, che aveva dedicato una attività incessante, con scritti, comizi e interventi di ogni tipo a favore della riforma, guidò in maniera altrettanto decisa anche la campagna elettorale del 1907. "Non vogliamo però politica ecclesiastica [...], ma popolare, economica specialmente.", troviamo scritto tra i suoi appunti autografi del periodo elettorale ${ }^{41}$. Le elezioni si conclusero, come è noto, con un enorme successo dei popolari trentini che conquistarono ben 7 seggi sui 9 assegnati al Trentino ${ }^{42}$.

\footnotetext{
${ }^{38}$ F. Rasera (come in nota 16), 432; G. Divina, Storia del Beato Simone da Trento. Trento 1902.

${ }^{39}$ Il contraddittorio Dr. Degasperi-Todeschini. Il Trentino (18 giugno 1906).

${ }^{40}$ Lueger nel movimento cristiano-sociale. Il Trentino (11 marzo 1910).

${ }^{41}$ Archivio De Gasperi, Austria II.

${ }^{42}$ Cfr. G. Vecchio (come in nota 7), 555.
} 
Anche la campagna elettorale per le elezioni suppletive del consiglio comunale di Trento nel 1910 fu condotta da De Gasperi, che era già stato eletto consigliere comunale l'anno precedente, introducendo avanzate tecniche di propaganda, come la proiezione di diapositive, l'uso di diagrammi, tabelle e cartine.

Il 30 marzo 1911 il presidente del Consiglio austriaco, il barone Bienerth, scioglieva il Reichsrat, accingendosi a governare temporaneamente al di fuori del controllo parlamentare, sulla base dell'articolo 14. A metà dell'aprile 1911 iniziò di fatto la campagna elettorale dei popolari trentini. De Gasperi, che aveva appena compiuto l'età di trent'anni, fu candidato, al posto di Bonfiglio Paolazzi, nel collegio di Fiemme e di Fassa, dove maggiore era la lotta gomito a gomito tra cattolici e volksbundisti. Malgrado le varie difficoltà incontrate, i popolari ottennero voti sufficienti per confermare in termini di seggi i risultati del 1907, inviando al Reichsrat Grandi, Delugan, Gentili, De Gasperi, Conci, De Carli e Tonelli, insieme al liberale Malfatti e al socialista Cesare Battisti. De Gasperi era stato eletto trionfalmente col $75 \%$ dei voti ${ }^{43}$.

Di fronte a De Gasperi e ai suoi collaboratori stava però il monito proveniente dall'amara esperienza dei cristiano-sociali viennesi, che dopo il successo del 1907, la morte di Lueger nel 1910 e la propria involuzione in senso conservatore, avevano proprio allora conosciuto un grave crollo di consensi, finendo nella capitale per essere scavalcati nel numero dei seggi complessivi dai socialdemocratici ${ }^{44}$.

Nel periodo che va dalle elezioni del 1911 allo scoppio della prima guerra mondiale, De Gasperi visse intensamente il proprio mandato elettorale $^{45}$. Al Reichsrat De Gasperi ricoprì la carica di membro della Commissione per il lavoro e di quella per la stampa (XXI sessione, 1911-1912). Fece inoltre parte della Delegazione austriaca. Dopo la riapertura del parlamento nell'estate del 1917 il suo carico di lavoro e la sua presenza alle riunioni delle varie commissioni aumentarono: fece parte della Commissione per il bilancio e fu membro sostituto della Commissione per l'alimentazione, per i contributi di sussistenza, per l'economia di guerra

${ }^{43}$ Cfr. ibidem, 577-578.

${ }^{44}$ Cfr. J. W. Boyer, Culture and Political Crisis (come in nota 13), 236-297.

${ }^{45}$ Cfr. R. Moscati, De Gasperi nel Parlamento austriaco, La nuova Antologia CXI (1976), 20-44; R. Schober, Alcide De Gasperi al parlamento a Vienna, in: De Gasperi e il Trentino tra la fine dell'800 e il primo dopoguerra, a cura A. CANAvero-A. Moioli. Trento 1985, 759-695; I. Ganz, La rappresentanza del Tirolo italiano alla Camera dei deputati di Vienna 1861-1914. Trento 2001, 228-238. 
e per i trattati. Fu rieletto membro della Commissione per la stampa e soprattutto fu membro della Commissione centrale per i profughi ${ }^{46}$.

Come deputato De Gasperi cercò di ottenere l'approvazione di specifici provvedimenti di legge a favore del Trentino. Lamentò il disinteresse del governo austriaco per i problemi economici del Trentino e per la mancata concessione di autonomia amministrativa ${ }^{47}$.

Nel corso della XXII legislatura, iniziata quando il giovane Carlo I riconvocò il parlamento che per tre anni era rimasto inattivo, De Gasperi concentrò la sua attività a favore dei profughi trentini, continuando in sostanza l'opera iniziata subito dopo l'ingresso in guerra dell'Italia, quando le autorità austriache, nutrendo forti dubbi sulla lealtà dei sudditi trentini, deportarono interi villaggi, disperdendone gli abitanti in varie zone dell'impero. 80.000 persone, circa un terzo degli abitanti della regione, dovettero abbandonare la propria terra e i propri averi ${ }^{48}$.

Per evitare l'invio al confino De Gasperi si recò a Vienna mettendosi a disposizione come delegato del Segretariato per richiamati e profughi e compiendo in tale veste viaggi di ispezione nella Boemia occidentale e in alcune regioni dell'Austria.

Da giovane deputato del parlamento viennese De Gasperi ebbe modo di vivere un'esperienza di cui conserverà poi un vivo ricordo anche da presidente del Consiglio italiano. Nel settembre del 1913 visitò il Palazzo della Pace internazionale all'Aia inaugurato un mese prima ${ }^{49}$.

Erano quegli anni in cui sembrava che il movimento pacifista e le aspirazioni a creare vincoli sopranazionali per evitare lo scoppio di conflitti fossero usciti dalla sfera dell'astratto e si fossero concretizzati sul piano giuridico nella ricerca di norme e istituti di diritto internazionale.

${ }^{46}$ Wien, Parlamentsarchiv, Abgeordnetenhaus des Reichsrates, Materie 34, Ausschussprotokolle.

${ }^{47}$ Cfr. il discorso tenuto da Gasperi sul rincaro dei prezzi il 13 ottobre 1911, in: StPAH, XXI. Session, 24. Sitzung, 918-920; inoltre il discorso sull'università italiana (come in nota 4 ).

${ }^{48}$ Cfr. in particolare il discorso di De Gasperi sull'assistenza ai profughi, 12 luglio 1917, in: StPAH, XXII. Session, 18 Sitzung, 915-919; sul bilancio dell'esercizio finanziario per il 1917/18 (durante il quale si soffermò ampiamente sulle condizioni del Trentino e sui soprusi delle autorità militari), 28 settembre 1917, in: StPAH, XXII. Session, 25. Sitzung, 1325-12329; sugli avvenimenti nel campo profughi di Wagna, 16 ottobre 1917, in: StPAH, XXII. Session, 29. Sitzung, 1482, $1524-1525$.

${ }^{49}$ Visitando il palazzo della giustizia internazionale. Il Trentino (27 settembre 1913), in: A. De Gasperi, I cattolici trentini sotto l'Austria (come in nota 14), vol. II, 378-382. 
La Corte di Arbitrato dell'Aia avrebbe dovuto essere un punto di avvio per un nuovo sistema di relazioni internazionali. Gli avvenimenti dei mesi successivi avrebbero invece dimostrato che quell'organo di giustizia, nato in un momento in cui le potenze erano ormai lanciate verso la guerra, era insufficiente.

De Gasperi aveva già da tempo avvertito i sempre più frequenti segni premonitori della crisi europea e l'insufficienza del sistema di rapporti internazionali allora in vigore. Senza ancora sviluppare proposte precise, arrivò tuttavia ad accennare alla necessità d'un sistema d'intesa e di collaborazione tra gli Stati, richiamandosi ad una suprema funzione arbitrale del papato come nel medioevo, oppure ad una suprema autorità politica sopranazionale. Nessuno può levare oggi una voce che imponga la pace, che richiami le coscienze al concetto della guerra giusta od ingiusta... Ma anche la concezione ghibellina del Medio Evo non ha oggi alcun raffronto. ... In vero il 'concerto europeo', la 'conferenza degli ambasciatori', la nuova organizzazione diplomatica potrebbero sostituire l'autorità dell'impero romano germanico, come l'invocava il grande Ghibellino, autorità civile somma e suprema che lasciava la massima autonomia alle nazioni ... ${ }^{50} \mathrm{Un}$ sistema di Stati integrati in un organismo politico comune, nel quale le singole nazionalità godessero però di garanzie e di libertà per autogovernarsi nelle proprie questioni specifiche. Questo modello di rapporti internazionali e interstatali sarebbe rimasto nella concezione politica di De Gasperi ben oltre gli anni qui considerati.

Le esperienze, le conoscenze e i contatti che De Gasperi aveva accumulato prima della Grande guerra, sarebbero poi stati ripresi e sviluppati agli inizi degli anni Venti a fianco di Luigi Sturzo nell'iniziativa del Partito Popolare Italiano di creare una "Internazionale Popolare", cioè un organismo di coordinamento dei partiti democratici cristiani europei che accogliesse le aperture internazionalistiche e le spinte al superamento dei nazionalismi dei leaders popolari dei vari paesi europei ${ }^{51}$. Ma ancora durante la guerra mondiale, nel febbraio del 1917, si tenne a Zurigo una conferenza di parlamentari e uomini politici provenienti dalla Svizzera, Germania, Austria, Ungheria e Polonia. Scopo dell'incontro era creare un'intesa organica fra leaders dei movimenti e delle organizzazioni cattoliche politiche, sociali e culturali delle varie nazioni, fondando una sorta

${ }^{50}$ Il turco ride. Il Trentino (9 luglio 1913), in: A. DE Gasperi (come in nota 14), vol. II, $375-377$.

51 Sull'iniziativa di Sturzo cfr. R. PAPINI, Il coraggio della democrazia. Sturzo e l'Internazionale popolare fra le due guerre. Roma 1995. 
di Unione internazionale cattolica, per presentarsi come una forza compatta dopo la cessazione delle ostilità e promuovere una ricostruzione europea sulla base di una Weltanschauung cristiana. La prima idea dell'iniziativa partì allora da un prominente parlamentare e politico cattolico italiano e da più parti si è avanzata l'ipotesi che si trattasse di Alcide De Gasperi ${ }^{52}$.

E' noto comunque che tutte queste iniziative non giunsero allora ad alcun risultato concreto. Sarebbero state però riprese dopo la seconda guerra mondiale e poi realizzate, il più delle volte dagli stessi politici che avevano contribuito al loro primo sviluppo molti anni addietro ${ }^{53}$.

${ }^{52}$ A. Matтiazzo, Le internazionali cattoliche: origini e programmi, in: Genesi della coscienza internazionalista nei cattolici fra ' 800 e '900. Padova 1983, 59-168.

${ }^{53}$ Cfr. Christdemokratie in Europa im 20. Jahrhundert, a cura di M. Genter-W. KaIser-H. Wohnout. Wien-Köln-Weimar 2001, in particolare 559-749. 